\title{
Late-onset coronary thrombosis and thrombocytosis following G-CSF treatment in a healthy donor: A Case Report
}

\author{
Cheng Xie ${ }^{1}$, Fan Xia ${ }^{1}$, Caiyun $\mathrm{Li}^{2}$, Haipeng Wang ${ }^{1}$, Bin Jiang ${ }^{1}$, Mianxian $\mathrm{Li}^{1}$, and Zexiong \\ Feng $^{1}$ \\ ${ }^{1}$ The First Affiliated Hospital of Soochow University \\ ${ }^{2}$ The Affiliated Suzhou Science and Technology Town Hospital of Nanjing Medical \\ University
}

November 17, 2020

\begin{abstract}
Granulocyte colony stimulating factor (G-CSF) has been widely used as a mobilizing agent to rapidly increase peripheral blood stem and progenitor cells. Limited data is available for G-CSF-associated late-onset coronary thrombosis and thrombocytosis. We report a case of a 23-year-old Chinese Han patient who presented with acute myocardial infarction and thrombocytosis after finishing bone marrow harvesting and peripheral blood stem cell collection following G-CSF treatment several days later. By using antiplatelet drugs and undergoing percutaneous transluminal coronary angioplasty, the patient's symptoms were relieved and his platelet level decreased to normal. This is the first suspected case report of late-onset coronary thrombosis and thrombocytosis related to G-CSF. Caution should be taken for the delayed adverse reactions in patients undergoing G-CSF mobilizing.
\end{abstract}

Late-onset coronary thrombosis and thrombocytosis following G-CSF treatment in a healthy donor: A Case Report

Fan Xia ${ }^{1 \#}$, Caiyun $\mathrm{Li}^{2 \#}$, Haipeng Wang ${ }^{3 \#}$, Bin Jiang ${ }^{3}$, Mianxian $\mathrm{Li}^{3}$, Zexiong Feng ${ }^{3^{*}}$, Cheng $\mathrm{Xie}^{{ }^{*}}$

1 Department of Pharmacy, the First Affiliated Hospital of Soochow University, Suzhou, 215006, China

2 Department of Pharmacy, the Affiliated Suzhou Science and Technology Town Hospital of Nanjing Medical University, Suzhou, 215153, China

3 Department of Cardiology, the First Affiliated Hospital of Soochow University, Suzhou, 215006, China

\# These authors contributed equally to this work.

*Corresponding author:

Cheng Xie, Department of Pharmacy, the First Affiliated Hospital of Soochow University, Suzhou, 215006, China; E-mail: xiecheng_1999@163.com.

Zexiong Feng, Department of Cardiology, the First Affiliated Hospital of Soochow University, Suzhou, 215006, China; E-mail:

dr_feng@126.com

\section{Abstract}


Granulocyte colony stimulating factor (G-CSF) has been widely used as a mobilizing agent to rapidly increase peripheral blood stem and progenitor cells. Limited data is available for G-CSF-associated late-onset coronary thrombosis and thrombocytosis. We report a case of a 23-year-old Chinese Han patient who presented with acute myocardial infarction and thrombocytosis after finishing bone marrow harvesting and peripheral blood stem cell collection following G-CSF treatment several days later. By using antiplatelet drugs and undergoing percutaneous transluminal coronary angioplasty, the patient's symptoms were relieved and his platelet level decreased to normal. This is the first suspected case report of late-onset coronary thrombosis and thrombocytosis related to G-CSF. Caution should be taken for the delayed adverse reactions in patients undergoing G-CSF mobilizing.

Keyworws : adverse reaction, late-onset, G-CSF, coronary thrombosis, thrombocytosis

Allogeneic hematopoietic stem cell transplantation is the preferred treatment for many malignant hematologic diseases. Granulocyte colony stimulating factor (G-CSF) has been widely used as a mobilizing agent to rapidly increase peripheral blood stem and progenitor cells (PBPC). Common adverse drug reaction (ADR) of G-CSF include bone and muscle pain, headache, fever, and inflammation at the injection site. Serious side effects include thrombosis and spleen rupture [1,2]. We describe the first suspected case of a 23-year-old man who presented late-onset coronary thrombosis and thrombocytosis after finishing bone marrow harvesting and peripheral blood stem cell collection following G-CSF treatment.

This is a 23-year-old Chinese Han patient just graduated from university. He was selected as a matched donor for his younger brother who had severe aplastic anemia (SAA). He was previously healthy and had no history of hypertension, diabetes mellitus, dyslipidemia, surgery, trauma, hematological disorders, psychosocial diseases or infectious diseases, but allergic to morphine. He denied family history of thrombotic diseases or heart diseases. He smoked 7-8 cigarettes per day for about 7 years. Clinical examination, electrocardiogram (ECG), coagulation markers, blood chemistry and blood test analysis results were normal. Serology was negative for hepatitis $\mathrm{B}$ and $\mathrm{C}$ viruses, and IgG was negative for cytomegalovirus and Epstein-Barr virus. He received G-CSF at $8.57 \mu \mathrm{g} / \mathrm{kg} /$ day (600 $\mu \mathrm{g}$ ) for 6 days by subcutaneous injection (from June 12, 2020 to June 17, 2020). Bone marrow harvesting and peripheral blood stem cell collection were respectively finished on June 16, 2020 and June 17, 2020.

However, the patient had persistent chest pain, toothache, profuse sweating and vomiting several times suddenly on June 23, 2020. Laboratory test showed high-sensitivity cardiac troponin T $1029 \mathrm{pg} / \mathrm{mL}$, myoglobin $328.7 \mathrm{ng} / \mathrm{mL}$, creatine kinase-MB $213.9 \mathrm{ng} / \mathrm{mL}$, white blood cell count (WBC) $17.68 \times 109 / \mathrm{L}$, red blood cell count $(\mathrm{RBC}) 3.85 \times 1012 / \mathrm{L}$, hemoglobin $(\mathrm{Hb}) 118 \mathrm{~g} / \mathrm{L}$ and platelets count (PLT) $160 \times 109 / \mathrm{L}$. ECG indicated ST segment elevation about $0.1-0.2 \mathrm{mV}$ in II, III and AVF leads. Echocardiography found weak segmental motion of the inferior and posterior wall of the left ventricular wall. ST-segment elevation myocardial infarction was diagnosed. Aspirin 300mg and ticagrelor 180mg were immediately taken. He underwent emergency percutaneous transluminal coronary angioplasty (PTCA), which revealed acute total occlusion of the middle right coronary artery and nearly normal left coronary artery without obvious lesion or plaque. Thrombelastography on July 2 revealed that inhibitory response to adenosine diphosphate $75.9 \%$, inhibitory response to arachidonic acid $75 \%$. Coagulation and platelet function test revealed that activated clotting time 138.4 sec, clot rate $56.8 \%$, platelet function 4.03. His PLT reached peak of $594 \times 109 / \mathrm{L}$ on July 4. Screening for autoimmune and blood diseases by the rheumatologist and hematologist were both negative. No further interventions were made to manage his thrombocytosis. He was discharged on July 8. The patient returned to the clinic for a blood test on July 24 and his PLT was dropped to $275 \times 109 / \mathrm{L}$. His recovery was uneventful. The blood test results of the patient throughout his disease course are shown in Figure 1.

G-CSF was the most possible factor which induced coronary thrombosis and thrombocytosis in this case. The patient in our paper was a presumably healthy young donor and only treated with G-CSF before coronary thrombosis. He had no cardiovascular risk factors such as hypertension, diabetes mellitus or dyslipidemia, and denied family history of thrombotic diseases or heart diseases. The probability of adverse drug reaction was assessed using the Naranjo algorithm [3]. A score of +7 was predictive of G-CSF being the culprit. 
The mechanism of thrombosis caused by G-CSF is unclear. The initial case of acute lower-extremity arterial thrombosis occurring in a patient with bladder cancer considered that an arterial thrombosis developed in partial relation to G-CSF-induced leukocytosis and a subsequent hypercoagulable state [4]. Subsequent research focused on the effects of G-CSF on the hemostatic system in healthy volunteers found a significant elevation in the maximum platelet aggregation rate induced by adenosine diphosphate (ADP) or collagen, thromboxane B2 level and amount of thrombin-antithrombin III complex [5]. After that, a randomized controlled trial (RCT) was performed to determine the effect of G-CSF on platelet aggregation and in vivo platelet activation. The results further confirmed G-CSF significantly enhanced ADP $(+40 \%)$, collagen $(+60 \%)$, arachidonic acid $(+75 \%)$-induced platelet aggregation and ristocetin-induced platelet aggregation $(+18 \%)$ without change on platelet count [6]. In addition, studies showed that enhanced platelet activation and aggregation could possibly contribute to serious cardiac adverse events in temporal relation to G-CSF administration [7-9]. However, the cases mentioned in above articles were patients had a history of coronary heart disease.

G-CSF could enhance platelet aggregation and activation in humans, as well as change of the count of platelets. Although thrombocytosis, thrombocytopenia or thrombosis caused by G-CSF are rare, close clinical monitoring including PLT should be checked during G-CSF treatment and a period after discontinuation. When G-CSF is administered to patient with a history of thrombosis, the prophylactic therapy with aspirin or P2Y12 antagonists should be considered depending on the clinical condition.

\section{RELATIONSHIP DISCLOSURE}

The authors declare nothing to report.

\section{Acknowledgments}

None.

\section{REFERENCES}

1. Michael A Pulsipher, Pintip Chitphakdithai, John P Miller, Brent R Logan, Roberta J King, J Douglas Rizzo, et al. Adverse events among 2408 unrelated donors of peripheral blood stem cells: results of a prospective trial from the National Marrow Donor Program. Blood. 2009. 113(15): 3604-11.

2. Kristina Hölig, Michael Kramer, Frank Kroschinsky, Martin Bornhäuser, Thilo Mengling, Alexander $\mathrm{H}$ Schmidt, et al. Safety and efficacy of hematopoietic stem cell collection from mobilized peripheral blood inunrelated volunteers: 12 years of single-center experience in 3928 donors. Blood. 2009. 114(18): 3757-63.

3. C A Naranjo, U Busto, E M Sellers, P Sandor, I Ruiz, E A Roberts, et al. A method for estimating the probability of adverse drug reactions. Clin Pharmacol Ther. 1981. 30(2): 239-45.

4. J A Conti, H I Scher. Acute arterial thrombosis after escalated-dose methotrexate, vinblastine, doxorubicin, and cisplatin chemotherapy with recombinant granulocyte colony-stimulating factor. A possible new recombinant granulocyte colony-stimulating factor toxicity. Cancer. 1992. 70(11): 2699-702.

5. M Kuroiwa, T Okamura, T Kanaji, S Okamura, M Harada, Y Niho. Effects of granulocyte colonystimulating factor on the hemostatic system in healthy volunteers. Int J Hematol. 1996. 63(4): 311-6.

6. Alexander O Spiel, Johann Bartko, Michael Schwameis, Christa Firbas, Jolanta Siller-Matula, Matthias Schuetz, et al. Increased platelet aggregation and in vivo platelet activation after granulocyte colonystimulating factor administration. A randomised controlled trial. Thromb Haemost. 2011. 105(4): 65562.

7. Y Fukumoto, T Miyamoto, T Okamura, H Gondo, H Iwasaki, T Horiuchi, et al. Angina pectoris occurring during granulocyte colony-stimulating factor-combined preparatory regimen for autologous peripheral blood stem cell transplantation in a patient with acute myelogenous leukaemia. Br J Haematol. 1997. 97(3): 666-8.

8. R Vij, D R Adkins, R A Brown, H Khoury, J F DiPersio, T Goodnough. Unstable angina in a peripheral blood stem and progenitor cell donor given granulocyte-colony-stimulating factor. Transfusion. 1999. 39(5): 542-3. 
9. Jonathan M Hill, Mushabbar A Syed, Andrew E Arai, Tiffany M Powell, Jonathan D Paul, Gloria Zalos, et al. Outcomes and risks of granulocyte colony-stimulating factor in patients with coronary artery disease. J Am Coll Cardiol. 2005. 46(9): 1643-8. 

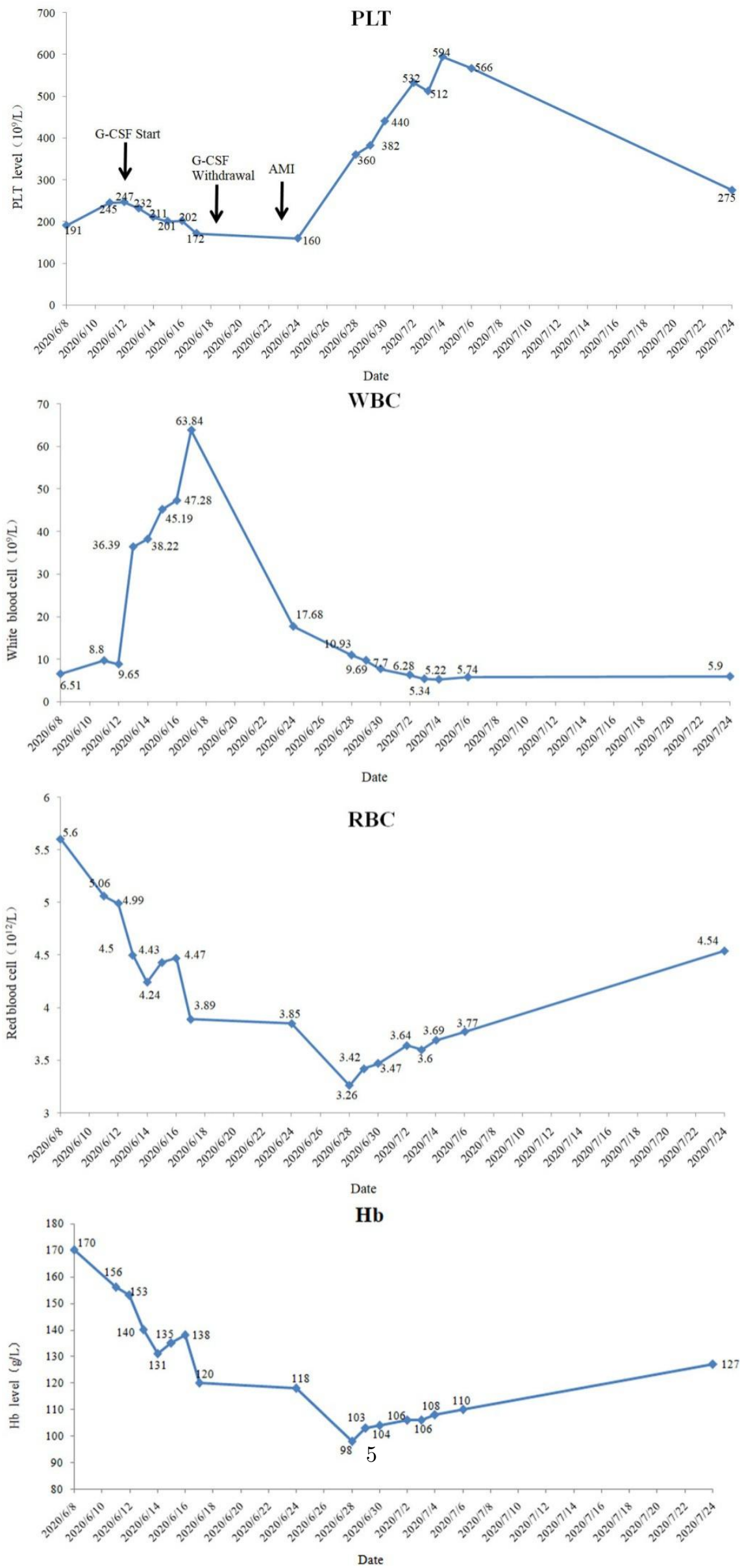
FIGURE 1 Serial changes of complete blood count during G-CSF therapy and withdrawal. GCSF, granulocyte colony stimulating factor; AMI, acute myocardial infarction; PLT, platelet; WBC, whiteblood cell; RBC, red blood cell; Hb, hemoglobin 